\title{
3 W Continuous-Wave Room Temperature Single- Facet Emission From Quantum Cascade Lasers Based On Nonresonant Extraction Design Approach
}

\section{Citation}

Lyakh, Arkadiy, Richard Maulini, Alexei Tsekoun, Rowel Go, Christian Pflügl, Laurent Diehl, Qi Jie Wang, Federico Capasso, and C. Kumar N. Patel. 2009. 3 W continuous-wave room temperature single-facet emission from quantum cascade lasers based on nonresonant extraction design approach. Applied Physics Letters 95(14): 141113.

\section{Published Version}

doi: $10.1063 / 1.3238263$

\section{Permanent link}

http://nrs.harvard.edu/urn-3:HUL.InstRepos:5343164

\section{Terms of Use}

This article was downloaded from Harvard University's DASH repository, and is made available under the terms and conditions applicable to Open Access Policy Articles, as set forth at http:// nrs.harvard.edu/urn-3:HUL.InstRepos:dash.current.terms-of-use\#OAP

\section{Share Your Story}

The Harvard community has made this article openly available.

Please share how this access benefits you. Submit a story.

Accessibility 


\title{
$3 \mathrm{~W}$ continuous-wave room temperature single-facet emission from quantum cascade lasers based on nonresonant extraction design approach
}

\author{
A. Lyakh, ${ }^{1}$ R. Maulini, ${ }^{1}$ A. Tsekoun, ${ }^{1}$ R. Go, ${ }^{1}$ C. Pflügl, ${ }^{2}$ L. Diehl, ${ }^{2}$ Q. J. Wang,${ }^{2}$ \\ Federico Capasso, ${ }^{2}$ and C. Kumar N. Patel ${ }^{1,3, a)}$ \\ ${ }^{1}$ Pranalytica, Inc., 1101 Colorado Ave., Santa Monica, California 90401, USA \\ ${ }^{2}$ School of Engineering and Applied Sciences, Harvard University, Cambridge, Massachusetts 02138, USA \\ ${ }^{3}$ Department of Physics and Astronomy, University of California, Los Angeles, \\ California 90095, USA
}

(Received 22 June 2009; accepted 3 September 2009; published online 9 October 2009)

A strain-balanced, InP-based quantum cascade laser structure, designed for light emission at $4.6 \mu \mathrm{m}$ using a new nonresonant extraction design approach, was grown by molecular beam epitaxy. Removal of the restrictive two-phonon resonant condition, currently used in most structure designs, allows simultaneous optimization of several design parameters influencing laser performance. Following the growth, the structure was processed in buried heterostructure. Maximum single-ended continuous-wave optical power of $3 \mathrm{~W}$ was obtained at $293 \mathrm{~K}$ for devices with stripe dimensions of $5 \mathrm{~mm} \times 11.6 \mu \mathrm{m}$. Corresponding maximum wallplug efficiency and threshold current density were measured to be $12.7 \%$ and $0.86 \mathrm{kA} / \mathrm{cm}^{2}$. (C) 2009 American Institute of Physics. [doi:10.1063/1.3238263]

Quantum cascade lasers (QCLs) are compact semiconductor light sources operating in the midinfrared spectral region (from $\sim 3$ to $20 \mu \mathrm{m}$ ) with high performance at room temperature. These devices are now commercially available and have a number of important military and civilian applications that broadly fall into two spectral bands, the midwave infrared (MWIR) and the longwave infrared (LWIR). The MWIR applications are highlighted by technologies for protecting aircraft from shoulder-fired missiles. In the LWIR range, most applications are related to various sensing problems, such as gas sensing for toxic industrial chemicals and explosives, as well as laser detection and ranging, and free space optical communications. Many of these applications would benefit from improved QCL performance, specifically higher output power and better wallplug efficiency (WPE), defined as the ratio between optical output power and electrical power. Optical power exceeding $1 \mathrm{~W}$ and WPE of approximately $10 \%$ in continuous wave $(\mathrm{cw})$ mode at room temperature has recently been reported for QCLs emitting at $4.6 \mu \mathrm{m} .{ }^{1-3}$ Higher optical power and WPE will lead to larger dynamic range, higher sensitivity, smaller size, and lower power requirements for systems incorporating these devices. Continued progress in this area strongly depends on how well different QCL structure design parameters influencing laser performance can be simultaneously optimized in a single structure. With a few exceptions most QCL structures are designed, today, using the two-phonon resonance approach $^{4}$ where an electron radiative transition from the upper laser level 4 to the lower laser level 3 is followed by two consecutive nonradiative transitions to the levels 2 and 1 (inset for Fig. 1). The energy spacings $E_{32}$ and $E_{21}$ are designed to be equal to or slightly larger than the longitudinal phonon (LO) energy $E_{\mathrm{LO}}(\sim 35 \mathrm{meV})$, leading to desirable very short lifetimes for electrons in the levels 3 and 2 . Since the energy spacing between the lower laser level and the lowest active region level $E_{31}$ is increased by factor of 2

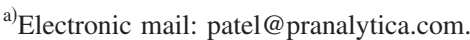

compared to the single-phonon resonance design, ${ }^{5}$ the twophonon resonance design has the advantage of lowering carrier population in the lower laser level 3 due to reduced carrier thermal backfilling into this state from the lowest active region state $1 .^{6}$ However, once the restrictive twophonon condition is met, there is not much flexibility remaining for changing other parameters of the design since active region layers thicknesses are essentially fixed by the resonance condition and the desired laser transition energy. In particular, it is difficult to increase energy spacing $E_{54}$ between the upper laser level 4 and the active region level 5 above it, a method to suppress parasitic carrier injection into the latter state, since level 5 is mostly localized in the same quantum wells as the low active region levels 2 and 1 .

One way of increasing the structure design flexibility is to remove the two phonon resonance condition, i.e., $E_{32}$ and $E_{21}$ both simultaneously being equal to $E_{\mathrm{LO}}$, without sacrificing the efficient carrier extraction from the lower laser

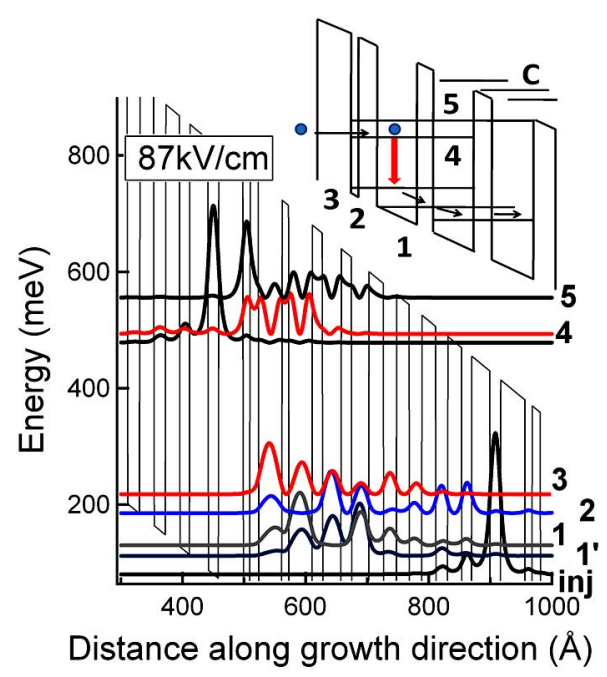

FIG. 1. (Color online) Band diagram of a QCL structure designed based on nonresonant extraction principle for light emission at $4.6 \mu \mathrm{m}$. Inset shows a schematic of a band diagram corresponding to two phonon design approach. 
level. The carrier lifetime $\tau_{i}$ for an energy level $i$ can be written as

$$
\frac{1}{\tau_{i}}=\sum_{f} \frac{1}{\tau_{i f}}
$$

where $\tau_{i f}$ is the scattering time from the energy level $i$ to a final level $f$ and the summation is over all possible final states. Each carrier transition time $\tau_{i f}$ reaches its minimum when energy spacing $E_{i f}$ is equal to $E_{\mathrm{LO}}$ and monotonically increases when $E_{i f}$ increases above $E_{\mathrm{LO}}$. Equation (1) suggests that the energy spacing $E_{21}$ can be increased substantially without increasing $\tau_{2}$ provided that there are at least two final levels 1 and $1^{\prime}$, instead of just one level 1 , for a transition from level 2. Significantly, carrier wave functions of these states, 1 and $1^{\prime}$, should have a large overlap with the wave function of the level 2. In this case, the summation over additional final states in Eq. (1), instead of just one, compensates for the increase in scattering times to individual final states due to increase in transition energies above $E_{\mathrm{LO}}$, and leads to lifetimes of lower states comparable to those achieved in two-phonon design. We named this design approach nonresonant extraction, or NRE. In analogous manner, the energy spacing $E_{32}$ can also be substantially increased without increasing $\tau_{3}$ provided that there are at least two final states 2 and $2^{\prime}$ for a transition from level 3. Removal of the two-phonon resonant condition makes the NRE approach a very attractive tool for QCL design. In this paper we present a description of a QCL structure designed using the NRE approach with corresponding record-high QCL experimental performance.

A conduction band diagram with the most relevant wave functions representing two gain stages of the structure is shown in Fig. 1. The active region of the structure is based on highly strained $(\sim 1 \%) \quad \mathrm{In}_{0.67} \mathrm{Ga}_{0.33} \mathrm{As} / \mathrm{Al}_{0.64} \mathrm{In}_{0.36} \mathrm{As}$ quantum wells and barriers with strain compensation in each stage. Doping level was empirically adjusted so that roll over current density of the optical power vs. current characteristic was approximately equal to $3 \mathrm{kA} / \mathrm{cm}^{2}$. As shown in Fig. 1, instead of a typical four-quantum well active region, characteristic for the two-phonon approach, we used a fivequantum well active region. This has two consequences. First, employment of a five-quantum well active region leads to the appearance of an additional active region level $1^{\prime}$, closely spaced with level 1 . Second, the energy spacing between the lower laser level 3 and the lowest active region level $1^{\prime}, E_{31}$, was increased up to $100 \mathrm{meV}$, further suppressing thermal back filling of the lower laser level 3. Both levels 1 and $1^{\prime}$ have a large overlap with level 2. Due to availability of two parallel transition for electron relaxation from the level 2, the calculated electron lifetime $\tau_{2}$ is only $0.34 \mathrm{ps}$, close to typical value for the two-phonon design, despite the fact that $E_{21}=55 \mathrm{meV}$ and $E_{21^{\prime}}=73 \mathrm{meV}$ are significantly above LO phonon energy. In addition, $\tau_{2}$ was not very sensitive to variation in active region layers thicknesses. This allows us, in addition to increasing $E_{31}$, to also increase $E_{54}$ up to $63 \mathrm{meV}$, while keeping high matrix element for the laser transition $z_{43}=1.48 \mathrm{~nm}$ and large upper laser level lifetime $\tau_{4}=1.77 \mathrm{ps}$. The parameters are calculated taking into account two consecutive stages under electric field equal to $87 \mathrm{kV} / \mathrm{cm}$. The laser emission wavelength is designed to be $\lambda=4.5 \mu \mathrm{m}$. This wavelength is of utmost importance since it is widely used for heat seeking missiles.

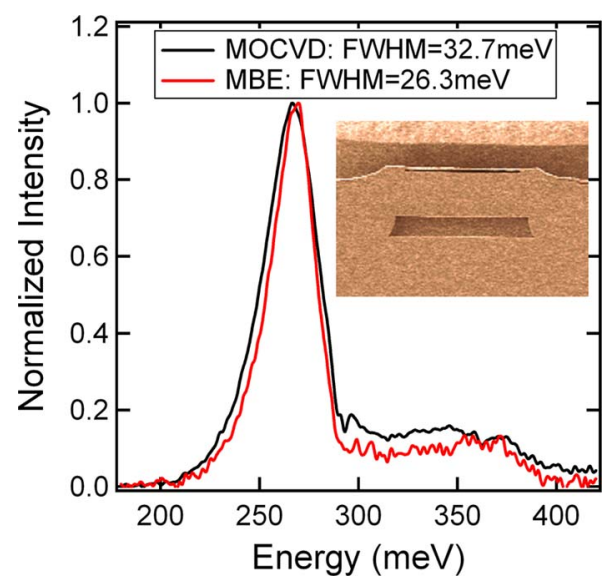

FIG. 2. (Color online) Electroluminescence spectra measured at $18 \mathrm{~V}$ for round mesas processed from MBE and MOCVD grown material with active region design shown in Fig. 1. MBE material demonstrated 20\% narrower spectrum (26.3 vs $32.7 \mathrm{meV}$ ). Inset shows $\mathrm{BH}$ overgrowth profile for RIE/ wet etching combination.

Preliminary experimental results for the structure described above, grown using metal organic chemical vapor deposition (MOCVD) and processed into buried heterostructure (BH) geometry using wet chemical etching, were reported in Ref. 1. In the present work, in addition to the description of the NRE concept and the band diagram of the structure, we also present new data, which show nearly a factor of 2 of improvement in optical power emitted by our QCLs, achieved using improved epigrowth and processing.

The QCL active region, along with the waveguide and contact layer sequence discussed in the previous work, ${ }^{1}$ was grown by molecular beam epitaxy (MBE).

Initially, part of the wafer was processed into round mesa devices for spectral electroluminescence characterization. Comparison between the electroluminescence spectra for MBE and MOCVD grown wafers is shown in Fig. 2. In addition to a slight blueshift of the peak, the MBE mesa demonstrated $20 \%$ reduction in linewidth (26.3 versus 32.7 $\mathrm{meV}$ ), which has direct impact on laser performance. First, differential gain is inversely proportional to the linewidth. Another contribution comes from nonresonant intersubband transitions, which can have high matrix element and, as a consequence, can significantly contribute to overall losses through "tails" of Lorentzian distribution. ${ }^{8,9}$ If the detuning between the resonance and the laser transition significantly exceeds the linewidth, these nonresonant losses are directly proportional to the linewidth. Therefore, a larger linewidth simultaneously increases nonresonant intersubband losses and reduces peak gain, both leading to higher laser threshold current density. The $20 \%$ improvement in linewidth for MBE-material should not be interpreted as inherent advantage for this growth method. Instead, the comparison between MOCVD and MBE-grown structures should be interpreted as a comparison between two growth runs under different conditions with different linewidth.

The wafer was then processed into a BH geometry. First, a combination of reactive ion etching and wet chemical etching was used to form the ridges, which were then overgrown with semi-insulating iron-doped InP. Resultant BH profile is shown in the inset of Fig. 2. After conventional substrate thinning, polishing, and episide/substrate metallization steps, the episide was electroplated with approximately $5 \mu \mathrm{m}$ thick 


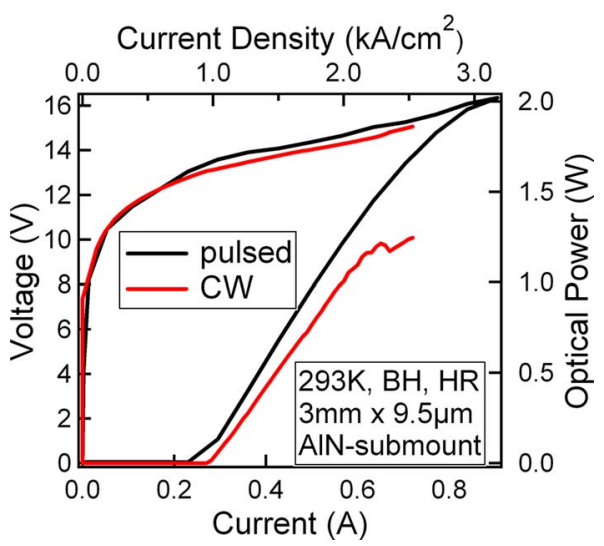

FIG. 3. (Color online) $\mathrm{cw}$ and pulsed power vs current and voltage vs current characteristics measured at $293 \mathrm{~K}$ for a $3 \mathrm{~mm}$ long, HR-coated laser chip with stripe width of $9.5 \mu \mathrm{m}$ mounted on AlN submount.

gold layer. The wafer was then cleaved into 3 and $5 \mathrm{~mm}$ long lasers that were subsequently high reflection (HR) coated. Finally, the chips were mounted episide down on aluminum nitride (AlN) and diamond submounts for pulsed and $\mathrm{cw}$ characterization. Pulsed testing was performed with $500 \mathrm{~ns}$ pulses and $0.5 \%$ duty cycle.

From dependence of threshold current density on reciprocal cavity length we found that the waveguide losses for these devices were $2.6 \mathrm{~cm}^{-1}$, reduced from $3.3 \mathrm{~cm}^{-1}$ measured in our previous work for MOCVD material. ${ }^{1}$ As discussed above, we believe that part of the reduction is due to lower nonresonant intersubband losses. Figure 3 shows a comparison between the pulsed and cw performance of a HR-coated $3 \mu \mathrm{mm} \times 9.5 \mu \mathrm{m}$ laser. Threshold current density, slope efficiency, and maximum WPE in pulsed/cw mode after correction for the measurement setup wiring resistance equal to $0.26 \mathrm{Ohm}$ (without correction) were measured to be $0.925 / 0.975 \mathrm{kA} / \mathrm{cm}^{2}, 4.1 / 3.3 \mathrm{~W} / \mathrm{A}$, and $15.4 / 13.0 \%$ (15.1\%/ $12.8 \%$ ), respectively. $13 \%$ is the highest WPE reported for single-ended emission for QCLs. Single-ended output is desirable, since it allows straightforward systems integration of high performance QCLs in practical applications. Ratio of peak power in pulsed mode to $\mathrm{cw}$ maximum power was found to be 1.6. This relatively small power degradation from pulsed to $\mathrm{cw}$ regime indicates good thermal performance of the fundamental quantum design, and efficient heat removal from the active region afforded by the $\mathrm{BH}$ geometry. Another growth run of the same nominal design, also processed in BH geometry, yielded devices with similar performance, indicating good design and process stability. Pulsed and $\mathrm{cw}$ power and voltage versus current characteristics for a $5 \mathrm{~mm}$ long HR-coated laser with $11.6 \mu \mathrm{m}$ stripe width, cleaved from the new wafer and mounted on a diamond submount using indium solder, are shown in Fig. 4. The maximum optical power in $\mathrm{cw}$ was measured to be $3 \mathrm{~W}$ at $293 \mathrm{~K}$, the highest value reported for QCL single-facet emission. After correction for the wiring resistance (without correction), maximum WPE was found to be $12.7 \%$ (12.4\%), close to the presented above $13 \%$ result for a $3 \mathrm{~mm}$ HR-coated laser mounted on AIN. Previously published best result for HR-coated QCL with dimensions $4 \mathrm{~mm}$ by $10.6 \mu \mathrm{m}$ mounted on diamond and also emitting at $4.6 \mu \mathrm{m}$ was 11.5 $\%$ at $293 \mathrm{~K}^{3}{ }^{3}$ In addition, the threshold current density and slope efficiency were measured to be $0.867 \mathrm{kA} / \mathrm{cm}^{2}$ and 3.3

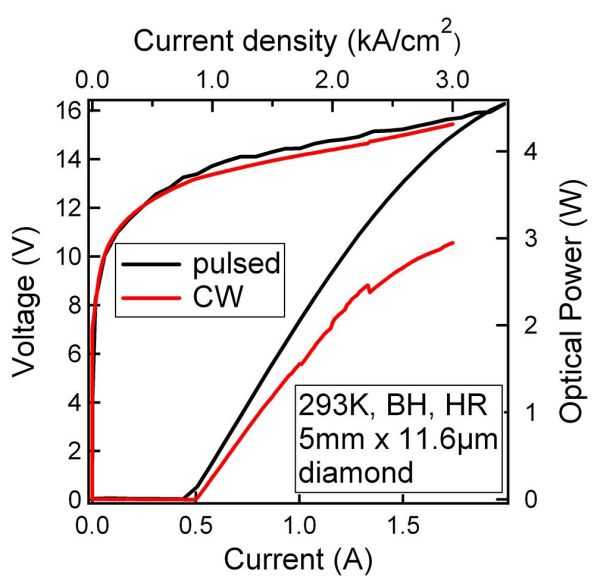

FIG. 4. (Color online) $\mathrm{cw}$ and pulsed power vs current and voltage vs current characteristics measured at $293 \mathrm{~K}$ for a $5 \mathrm{~mm}$ long, HR-coated laser chip with stripe width of $11.6 \mu \mathrm{m}$ mounted on diamond submount.

W/A, respectively. Ratio of maximum power in pulsed to $\mathrm{cW}$ modes was found to be 1.53. Electromigration of indium solder used for laser bonding to the diamond submounts significantly reduces laser lifetime. Therefore, indium/diamond combination cannot be used in commercial applications. However, such results are of significant scientific and technical interest since diamond has the highest conductivity among all known materials, and therefore, the data show the upper limit for $\mathrm{cw}$ performance.

In conclusion, we presented a new QCL design approach based on nonresonant extraction that permitted us to achieve record-high continuous wave QCL performance at room temperature. We showed that this approach offers more design flexibility compared to the traditional two-phonon design. $\mathrm{cw}$ room temperature WPE of $13.0 \%$ and maximum power of $3 \mathrm{~W}$ for single-facet emission were measured for QCLs grown by $\mathrm{MBE}$ and processed into $\mathrm{BH}$ using reactive ion etching/wet etching combination.

This work was supported in part through a DARPA Contract No. W911QX-07-C-0041 (approved for public release, Distribution Unlimited). The work at Harvard University was performed in part at the Center for Nanoscale Systems, a member of the National Nanotechnology Infrastructure Network, which is supported by the National Science Foundation under NSF Award No. ECS-0335765.

${ }^{1}$ A. Lyakh, C. Pflügl, L. Diehl, Q. J. Wang, F. Capasso, X. J. Wang, J. Y. Fan, T. Tanbun-Ek, R. Maulini, A. Tsekoun, R. Go, and C. K. N. Patel, Appl. Phys. Lett. 92, 111110 (2008).

${ }^{2}$ Y. Bai, S. R. Darvish, S. Slivken, W. Zhang, A. Evans, J. Nguyen, and M. Razeghi, Appl. Phys. Lett. 92, 101105 (2008).

${ }^{3}$ Y. Bai, S. Slivken, S. R. Darvish, and M. Razeghi, Appl. Phys. Lett. 93 021103 (2008)

${ }^{4}$ D. Hofstetter, M. Beck, T. Aellen, J. Faist, U. Oesterle, M. Ilegems, E. Gini, and H. Melchior, Appl. Phys. Lett. 78, 1964 (2001).

${ }^{5}$ J. Faist, F. Capasso, D. Sivco, C. Sirtori, A. Hutchinson, and A. Cho, Science 264, 553 (1994).

${ }^{6}$ J. Faist, D. Hofstetter, M. Beck, T. Aellen, M. Rochat, and S. Blaser, IEEE J. Quantum Electron. 38, 533 (2002).

${ }^{7}$ J. Faist, F. Capasso, D. Sivco, A. Hutchinson, C. Sirtori, S. Chu, and A. Cho, Appl. Phys. Lett. 65, 2901 (1994).

${ }^{8}$ A. Lyakh, R. Maulini, A. Tsekoun, R. Go, and C. K. N. Patel, Appl. Phys. Lett. 92, 211108 (2008).

${ }^{9}$ A. Wittmann, T. Gresch, E. Gini, L. Hvozdara, N. Hoyler, M. Giovannini, and J. Faist, IEEE J. Quantum Electron. 44, 36 (2008). 